\title{
Oxytocin modulates social brain network correlations in resting and task state
}

\author{
Qingyuan $\mathrm{Wu}^{a}$, Qi Huang ${ }^{a}$, Chao Liu ${ }^{a *}$, Haiyan $\mathrm{Wu}^{b *}$ \\ aState Key Laboratory of Cognitive Neuroscience and Learning \& IDG/McGovern Institute for Brain Research, \\ Beijing Normal University, 100875, Beijing, China \\ ${ }^{b}$ Centre for Cognitive and Brain Sciences and Department of Psychology, University of \\ Macau, Macau, China \\ E-mail: liuchao@bnu.edu.cn(C.L); haiyanwu@um.edu.mo(H.W)
}

\begin{abstract}
Oxytocin (OT) is a neuropeptide that modulates social behaviors and the social brain. The effects of OT on the social brain can be tracked by assessing the neural activity in the resting and task states, providing a system-level framework for characterizing state-based functional relationships of its distinct effect. Here, we contribute to this framework by examining how OT modulates social brain network correlations during the resting and task states using fMRI. Firstly, we investigated network activation, followed by analyzing the relationship between networks and individual differences measured by the Positive and Negative Affect Schedule and the Big-Five scales. Subsequently, we evaluated functional connectivity in both states. Finally, the relationship between networks across the states was represented by the predictive power of networks in the resting state for task-evoked activity. The difference in predicted accuracy between subjects displayed individual variations in this relationship. Our results showed decreased dorsal default mode network (DDMN) for the OT group in the resting state. Additionally, only in the OT group, the activity of the DDMN in the resting state had the largest predictive power for task-evoked activation of the precuneus network (PN). The results also demonstrated OT reduced individual variation of PN, specifically, the difference of accuracy between predicting a subject's own and others' PN task activation. These findings together suggest a distributed but modulatory effect of OT on the association between resting brain networks and task-dependent brain networks, showing increased DDMN to PN connectivity after OT administration, which may support OT-induced distributed processing during task performance.
\end{abstract}

Keywords: oxytocin, fMRI, face, self-resemblance, social brain, self-other overlap

\section{Abbreviations}

OT, oxytocin; PL, placebo; fMRI, functional magnetic resonance imaging; rsfMRI, resting-state fMRI; BOLD, blood-oxygenlevel-dependent; MPFC, medial prefrontal cortex; ACC, anterior cingulate cortex; 
TPJ, temporal-parietal junction; PCC, posterior cingulate cortex; ICA, independent component analysis; ICs, independent components; RSNs, resting-state networks; DMN, default mode network; VDMN, ventral default mode network; DDMN, dorsal default mode network; PN, precuneus network; BGN, basal ganglia network; LECN, left executive control network; RECN, right executive control network; PN, precuneus network; SN, sensorimotor network; ASN, anterior salience network; AN, auditory network; HVN, higher visual network; LN, language network; PSN, posterior salience network; PVN, primary visual network; PANAS, Positive and Negative Affect Schedule; SM, self-model; OM, other-model.

\section{Introduction}

Human social life, from processing self-information to generating impressions and maintaining social relationships with others, requires social functions in the brain. Distinct personality traits probed by different scales have been associated with different performance in social function, and the difference in neural mechanisms among the variety of traits has been discussed[1, 2, 3]. Critically, social functions are supported by the social brain network, which may comprise individual differences, especially for different states[4, 5]. For example, in the resting state, individual function networks may deviate from the group map and indicate variability across subjects[5, 6]. Compared to the resting state, external stimuli in a task state modulate the connectivity of these brain networks[7]. In recent years, researchers have gradually realized that the patterns of brain activity exhibited by individuals at rest could be a critical scaffold for people to perform behaviorally relevant tasks[8, 9, 10, 11, 12]. As the general functional connectivity accounting for the resting-state and task-based fMRI drives reliable and heritable individual differences in functional brain networks [13], exploring individual differences with a combination of brain networks across states and how those differences are affected are emerging areas in neuroscience.

Oxytocin (OT) has been commonly used to modulate human social behavior and neural activity of the social brain in both resting and task states[14,15,16,17, 18, 19, 20]. The social brain is a set of regions, including the medial prefrontal cortex (MPFC), anterior cingulate cortex (ACC), the temporal-parietal junction (TPJ), inferior frontal gyrus, and anterior insula [21, 22, 23, 24]. Some task-dependent studies revealed how OT impacts some of these brain regions. For example, the stress-induced response of the ACC is reduced by 0T[25]. Compared to the placebo (PL) group, the activation of the MPFC and ACC is also inhibited when patients with generalized social anxiety disorder see an emotional face in the OT group[26]. With regard to the resting state, several studies have found that OT changes the activation of networks consisting of regions that belong to the 
social brain $[27,28,29]$. Zheng et al. reported that OT leads to lower synchronization in the default mode network (DMN), which includes the posterior cingulate cortex (PCC), precuneus, MPFC, and TPJ $[27,28]$. Another research found that the effective flow from the midline default network (including the PCC and precuneus) to the salience network (including the ACC and insula) is increased by 0T[29].

To the best of our knowledge, most of the previous studies have generally investigated the effects of OT on the resting- or task-state brain network separately at the group level. However, the question regarding the mechanism by which OT impacts the scaffold effect of the networks' spontaneous activity during the resting state remains unknown. A previous study indicated that resting-state fMRI (rsfMRI) predicts individual differences in brain activity during task performance[9]. Subsequent studies confirmed the prediction from rsfMRI to task state fMRI [30, $31,9,32]$. However, a previous study also suggested that the brain network organization during a task is distinct from that in the resting state[33]. Given the individual variations in resting-state networks (RSNs), their influence on the brain network in subsequent tasks may be state-dependent. Although many studies have suggested that OT affects human social behavior and brain activity[34, $35,36,37]$, the lack of a cross-state brain network investigation makes it impossible to reveal the mechanism of OT's modulation effect on the link between task-free and task-dependent brain networks.

On the other hand, studies have highlighted subdivisions of classical brain networks in recent years. For instance, DMNs have been divided into two sub-networks: the ventral default mode network (VDMN) and dorsal default mode network (DDMN)[38, 39, 40]. The VDMN is linked to the association with memory-based reconstruction, while the DDMN is related to introspection about mental states and the evaluation of emotional valence[41, 42]. Additionally, the precuneus network (PN), including the precuneus, middle cingulate cortex, posterior inferior parietal lobule, and dorsal angular gyri, has been argued to be independent of the well-known DMN[43, 44]. Although the impact of OT on the DMN and the brain regions belonging to it has been discussed in many studies $[27,28,29]$, the specific modulatory effects of OT on the connectivity among social subnetworks are largely unknown.

To investigate this research question, we tested the modulatory effects of OT on bloodoxygenlevel-dependent (BOLD) signals during the resting state and a face perception task. Independent component analysis (ICA) was used to extract the features in both states at the network level[45, 32]. After the networks were identified from both groups during task-dependent and resting states, we compared the correlations between networks across the states. This allowed us to probe the critical question regarding the effect of OT on resting-task brain network correspondence. We aim to address the following three key hypotheses in this work: 1) OT can decrease the DMN's sub-network activity and change its connectivity with other brain networks in both states; 2) OT can influence resting-task brain network correspondence, particularly for brain 
regions related to the social brain networks; and 3) OT may make human behavior patterns more consistent by reducing self-other differences in the resting-task correspondence.

\section{Method}

\subsection{Participants}

Fifty-nine right-handed male participants (age range $20.9 \pm 2.32$ years) were recruited via an online recruiting system. All participants had 13-18 years of education. They completed the screening form, and those who confirmed that they had no significant medical or psychiatric illness, were not using any medication, and were not drinking and/or smoking daily were included in the study. Smoking or drinking (except water) was prohibited for 2 hours before the experiment. The participants provided written informed consent before each experiment and received full debriefing on the completion of the experiment. The study was approved by the local ethics committee of Beijing Normal University.

\subsection{Rest and Task data sets}

We used the resting and task state data sets collected from the same participants who were students at the Universities of Beijing and who had been undergoing OT (OT group) or PL (PL group) manipulation. 


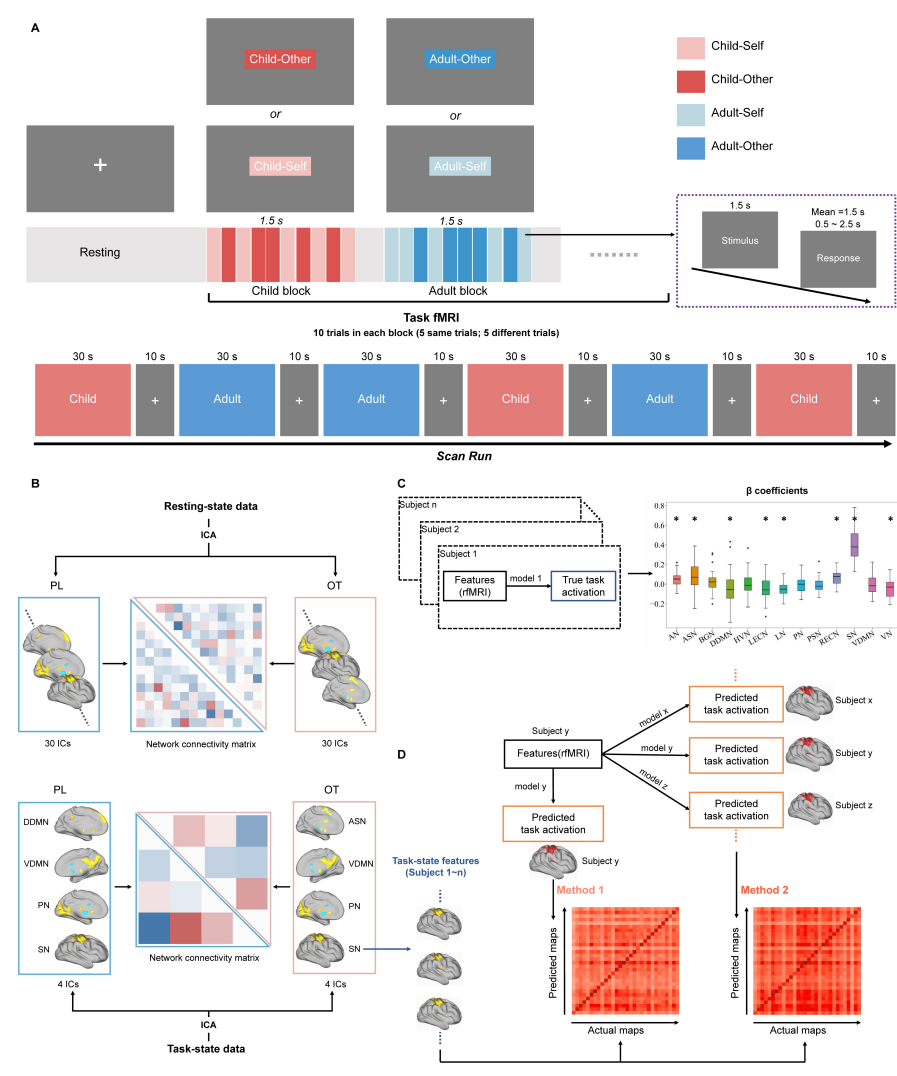

Figure 1: Diagrams representing our methods. A) Task procedure. Materials in the child block were morphed using a stranger child's face with the participant's (Self) face or another 23-year-old male's (Other) face. Materials in the adult block were morphed using a stranger adult's face and the participant's (Self) face or 23-year-old male's (Other) face. B) Feature extraction. We selected 30 features from the resting-state data and four features from the task state data from both groups. The correlation matrix was calculated for both states. C) Construction of the prediction models. In the training model step, we predicted features in task state by features in resting state and constructed a generalized linear model for each subject in this process. The outcomes were beta coefficients of each regressor (resting-state features). The beta values of features belonging to one network were averaged to be the network's predictive power. D) The prediction model testing. We calculated the fitness between predicted task activation and the actual task activation for testing models. The first method to gain the prediction maps used one subject's features (ICs extracted from rsfMRI data) and his own model. For this procedure, all participants shared one prediction map. The second method used one participant's features (ICs extracted from rsfMRI data) and the model of others to generate a prediction map for each participant.

For the resting data set, we used fMRI data similar to the resting-state data that have been published for resting-state network studies only[16, 27, 28]. For the task data set, we used the task fMRI data that have been used for task fMRI analyses only [17], which provided the task descriptions. Specifically, we used a face perception task with morphed self and other faces[17]. Four types of face stimulus were generated: child-self (morphing the participant face with one of two 1.5-year-old child's face with a neutral expression), child other (morphing the child's face with 
one of two 23-year-old male faces), adult-self, and adult-other. Each participant completed two runs. Each run included three adult blocks and three child blocks in a randomized sequence. Ten trials were conducted in each block. The participants were asked to recognize whether the generated face was similar to their own face (Figure 1A).

\subsection{Materials}

All participants had taken their frontal-face images with a neutral expression 3 days before the scanning day. The morphed faces of four experimental conditions were created based on these images and one of the two adult faces with a neutral expression (a 23-year-old male face) or one of the two 1.5-year-old child faces. We used Adobe Photoshop CS to standardize the photograph to black and white and excluded all features except the interior characteristics of the face. Then, we created the stimuli with 50/50 morph of the two selected faces using the Abrosoft Fanta Morph (www.fantamorph.com) software[46, 47, 48]. Thirty calibration locations were used to transform the morphed face into a standard face space, and all output morphed faces were resized to $300 \times 300$ dpi. All pictures were presented on a 17-inch Dell monitor with a screen resolution of $1024 \times 768$ pixels and $60 \mathrm{~Hz}$ refresh frequency, the visual angle of the face images is $4.3^{\circ} \times 4.6^{\circ}$ and the mean luminance of stimulus was $166 \mathrm{~cd} / \mathrm{m}^{2}$.

\subsection{MRI data acquisition}

All images were acquired on a 3T Siemens Tim Trio scanner with 12-channel head coil. Functional images employed a gradient-echo echo-planar imaging (EPI) sequence with following MRI scanner parameters:(40ms TE, $2 s$ TR, 90 flip, 210mm FOV, 128 by 128 matrix, 25 contiguous $5 \mathrm{~mm}$ slices parallel to the hippocampus and interleaved). We also acquired from all participants whole-brain T1-weighed anatomical reference images $\left(2.15 \mathrm{~ms}\right.$ TE, $1.9 \mathrm{~s}$ TR, $9^{\circ}$ flip, 256mm FOV, 176 sagittal slices, $1 \mathrm{~mm}$ slice thickness, perpendicular to the anterior-posterior commissure line).

2.5. fMRI data preprocessing fMRI data preprocessing was performed using Statistical Parametric Mapping software (SPM12: Wellcome Trust Centre for Neuroimaging, London, UK). The functional image time series were preproessed to compensate for slice-dependent time shifts, motion corrected, and linearly detrended, then coregistered to the anatomical image, spatial normalized to Montreal Neurological Institute (MNI) space and spatially smoothed by convolution with an isotropic Gaussian kernel (FWHM=6 mm). The fMRI data were high-pass filtered with a cutoff of $0.01 \mathrm{~Hz}$. The white matter (WM) signal, cerebrospinal fluid (CSF) signal and global signal, as well as the 6-dimensional head motion realignment parameters, the realignment parameters squared, their derivatives, and the squared of the derivatives were regressed. The resulting residuals were then low-pass filtered with a cutoff of $0.1 \mathrm{~Hz}$. 


\subsection{Feature extraction}

ICA was conducted using the Group ICA fMRI Toolbox (GIFT) to extract the brain activation features[49]. For rsfMRI data, in the first step, GIFT estimated the number of independent components (ICs) to be extracted from the preprocessed rsfMRI data. According to this estimated number, we obtained 36 ICs from rsfMRI data in the OT group and 35 ICs in the PL group. Second, we calculated the correlation coefficients between the ICs and RSN templates[10]. These templates included 14 brain networks(SI Figure 3): the basal ganglia network (BGN), visuospatial network (VN), right executive control network (RECN), VDMN, DDMN, sensorimotor network (SN), anterior salience network (ASN), auditory network (AN), higher visual network (HVN), left executive control network (LECN), language network (LN), PN, posterior salience network (PSN), and primary visual network (PVN)[40]. Each IC was compared with all brain network templates and labeled based on the most similar network defined in the template. Finally, 30 ICs with correlation coefficients greater than 0.1 were separately retained as predictors for both groups (Figure 1B) $[10]$.

For the task-state fMRI data, after estimating and analyzing them using the GIFT, 31 ICs were obtained in the OT group and 30 ICs in the PL group. Next, the time courses of these ICs were correlated with the modeled time course (the beta maps) for all groups in the task of the 1st level SPM.mat. The selected regressors were "self-child*bf(1)", "other-child*bf(1)", "self-adult*bf(1)", and "other-adult*bf(1)" time courses[49]. ICs that were most related to task activation (correlation coefficient > 0.7) were chosen as features. Finally, four ICs were chosen as features in both the PL and OT groups (Figure 1B). Then we labeled them using the template.

\subsection{Predictive Model}

To predict the task-state fMRI by rsfMRI, following the group ICA described above, we used spatial-time regression to reconstruct the subject-specific spatial z-score maps of each IC during both states. For each subject, all ICs extracted from the rsfMRI were applied to predict each IC of the task-state fMRI data. Therefore, we constructed four multiple linear regression models for each subject; every model included 30 regression coefficients[9]. The coefficients of ICs belonging to the same network template were averaged to intuitively observe each RSN's predictive power (Figure 1C). Then, we used a one-sample t-test to assess whether the RSNs activation could significantly predict the task-state brain network activation.

We also examined the predictive effect of individual differences in psychometric scale scores on the activation of RSNs. First, we computed the number of ICs contained in each network of each subject at rest. An independent sample t-test was performed on the number of ICs contained in the same network in the OT and PL groups. This allowed us to compare the activity of individual brain networks in different groups[50]. Previous studies have indicated that the effect of OT on brain activity is modulated by emotional state and individual variability[51, 34, 52]. Thus, we measured whether OT could affect the associations between brain network activation and subjective emotional states as well as personality traits. Emotional states were measured using the Positive 
and Negative Affect Schedule (PANAS), and the Big-Five scale was used to detect the participants' personality traits. Regression analysis was performed using generalized estimated equations to predict the number of ICs in the specific networks associated with social brain networks, including the BGN, RECN, VDMN, DDMN, SN, ASN, LECN, and PN. The predictors in the generalized estimated equations were the PANAS and Big-Five scores measured after OT and PL manipulation[50].

\subsection{Functional connectivity analysis}

We also analyzed the relationship between networks in both the resting and task states. ICs sharing the same label were averaged at the voxel level to indicate the brain network activation. We then calculated the Pearson coefficient matrices of all networks for both groups in the two states. Because the two groups of extracted ICs differed in the task state, the independent t-test was only performed to probe the influence of OT on the network correlation at rest. P-values were adjusted using Benjamini and Hochberg's FDR correction for multiple comparisons [50].

\subsection{Model test}

The coefficient of determination was regarded as an indicator for estimating the predictive power of our models and was compared with the null model (all regressors were equal to 1). Then we performed a one-sample t-test for the indicators of each model and assessed the performance of the models.[53].

One way to assess the model performance was to obtain the prediction accuracy by comparing the actual task maps with the prediction activation maps. The prediction activation maps could be derived using two methods. The first method combined the rsfMRI data of subject X with his own model (Self-model, SM), and comparing this prediction map with all subjects' actual task maps[9]. The second method combined the rsfMRI data of subject X with the model of subject Y (Othermodel, OM) to obtain a prediction map for subject Y. In other words, every subject had a specific prediction map in this method. The correlation coefficients between prediction maps and the actual task-state activity maps were considered as the predicted accuracy (Figure 1D). Each subject's RSNs activation was used to predict the task-related brain activation in all subjects. A Pearson correlation matrix was obtained for each IC (Figure 1D). The diagonal elements indicate the selfpredicted accuracy (X's resting brain activation predicts X's task brain activation), and the extradiagonal elements indicate the other-predicted accuracy (X's resting brain activation predicts Y's task brain activation). Thus, the difference between the two accuracies represent the individual variance (self-other difference). The percentage of the self-other difference relative to the average of other-predicted accuracy could indicate the relative size of the difference[9]. We then compared the percentage of self-other differences between the PL and OT groups using an independent sample t-test. 


\section{Results}

\subsection{The effect of OT on the resting brain networks is modulated by individual differences}

We first examined the effect of OT on brain network activity at rest. Networks correlated with the emotional or social brain (ASN, BGN, DDMN, LECN, RECN, SN, PN, and VDMN) were selected for the following analysis. The average number of ICs that belong to brain networks was used to represent each network's activation during the resting state. This was applied as the indices to measure OT's impact. The results showed a decreased number of ICs in PN $(t(56)=-3.6, p<0.001)$ and DDMN $(\mathrm{t}(56)=-2.84, p=0.006)$, but increased the number of ICs belonging to BGN $(t(56)=$ $11.59, p<0.001)$ and LECN $(t(56)=2.7, p=0.009)$ in the OT group. Meanwhile, there was no significant effect on ASN $(t(56)=-1.9, p=0.063)$, $\operatorname{SN}(t(56)=1.26, p=0.214)$, VDMN $(t(56)=1.78$, $p=0.081)$, and $\operatorname{RECN}(t(56)=1.25, p=0.218$, Figure $2 \mathrm{~A})$.

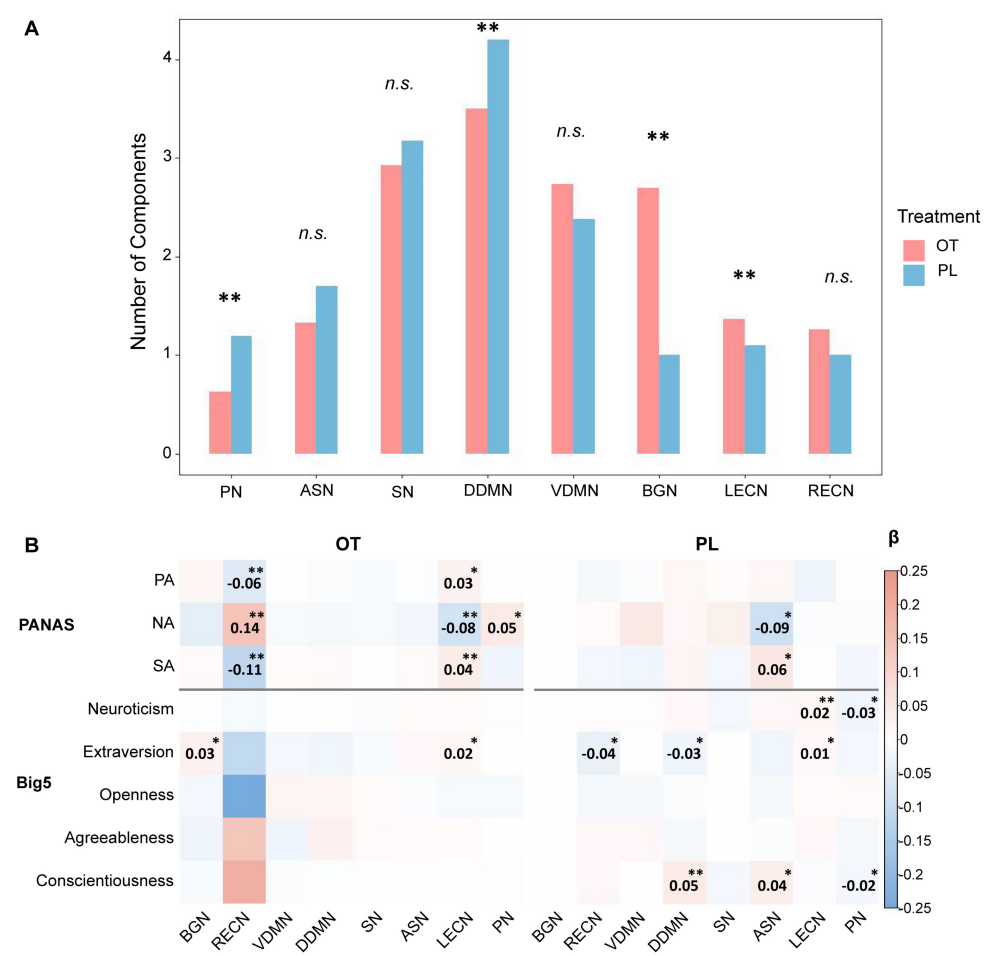

Figure 2: PANAS and Big-Five scores' associations with brain networks across the two groups. A) The average number of ICs in PN and DDMN were decreased by OT, while BGN and LECN were increased by OT. B) Beta values of generalized estimated equations-based associations between the occurrence of the ICs of interested network and scale scores, including affective scores (positive (PA) and negative (NA)) and personality scores. ${ }^{*} p<0.05,{ }^{* *} p<0.01$.

To test whether the emotional and personal traits were correlated with brain activity, we measured the scale scores after drug administration. A significant effect of OT on the presentation 
of emotion and personality in brain networks may indicate that the subjective affective rating scores (measured by PANAS) and personality scores (measured by Big-Five) could predict the occurrence of brain networks. The results showed approximately all the emotional scores were associated with bilateral executive control network (ECN) activity, but only the extraversion scores on the personality scale could predict BGN and LECN activity in the OT group. However, for the PL group, the personality scores had significant predictive effects on multiple brain networks, while emotional scores were almost completely unrelated to the activity of brain networks(Figure 2B).

\subsection{Network correlation in resting and task state}

Next, we explored the influence of OT on the relationship between brain networks by calculating the functional connection matrix in the two states respectively. The results showed that OT not only affected the connection strength but also the direction of correlation between brain networks in the resting state (see SI Figure 1, and Table 1). To better visualize the effect of OT, we compared the matrix of the OT group with that of the PL group (Figure 3A and Table 1).

A

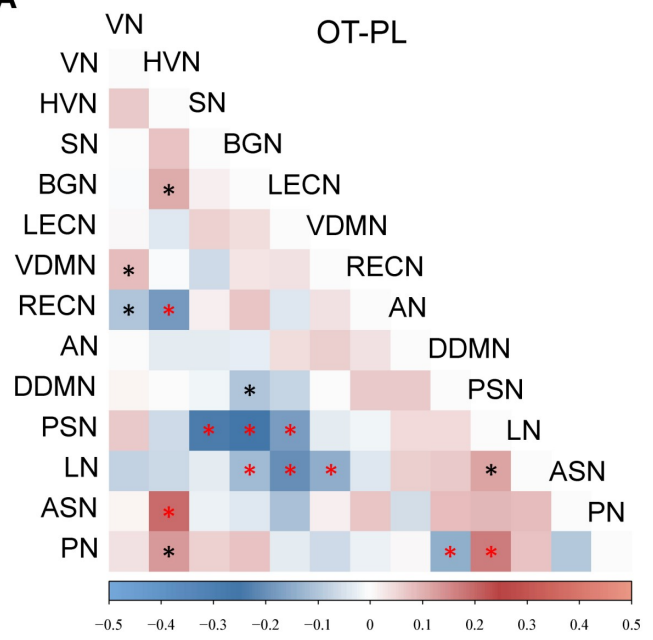

B

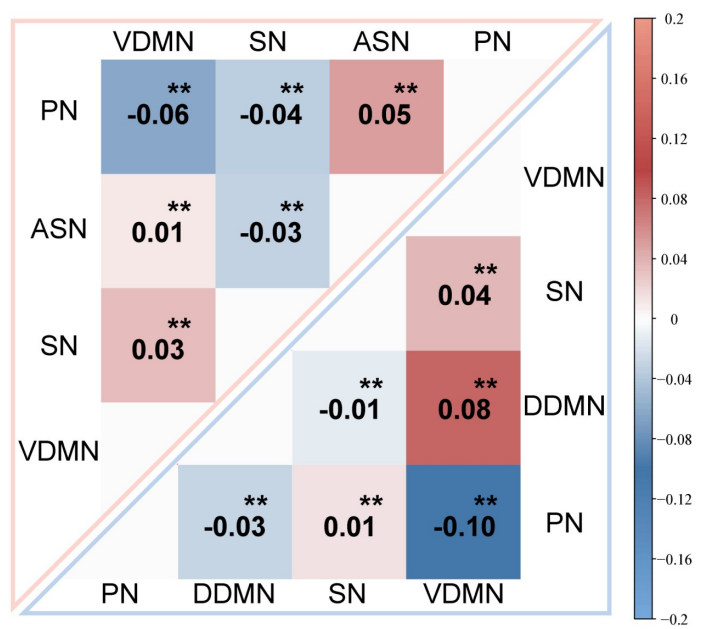

Figure 3: The difference of correlation matrix between brain networks (OT-PL). A) The difference between two grouplevel matrixes. The red star indicates $p<0.001$, and the black star indicates $p<0.05$. B) The task state brain network correlation matrix. Group-level correlation matrix of features extracted from the task fMRI data. $p_{F D R}$ adjustment for multiple comparisons. ${ }^{* *} p<0.01$

For the task-state dataset, the ICs correlated to the task were labeled as PN, SN, ASN, and VDMN in the OT group and those for the other group were labeled as PN, SN, DDMN, and VDMN. The result showed a difference about the activity of DDMN between groups, but on difference for VDMN. This finding was consistent with the resting-state results, which further indicated that VDMN and DDMN may affected differently by OT. Furthermore, we explored the task-state brain network correspondence. The results indicated that the correlation between PN and ASN was opposite to 
the correlation between PN and DDMN (Figure 3B). This is distinct from the pattern observed during the resting state. At that state, the correlation between PN and ASN was the same as the correlation between PN and DDMN in both groups (SI Figure 1).

\subsection{Associations between resting-state and task-state networks}

Figure 4 shows the contribution of resting ICs in recovering the activation of task ICs. All brain networks in the task state, except for PN in the OT group, could be best predicted themselves in

Table 1: RSNs correlation coefficients

\begin{tabular}{llccc}
\hline \hline & OT & \multicolumn{3}{c}{ PL t-score } \\
\hline
\end{tabular}

Note: Means, and two-tailed t-test (direction: OT - PL) results of the comparisons on functional connectivity in the resting state.

the resting state. In the PL group, the strongest predictor of PN in the task state was PN in the resting state. However, under the influence of OT, the predictive power of DDMN was larger than that of PN itself $\left(\right.$ beta $_{D D M N}=0.56$, beta $_{P N}=0.26, t(118)=3.00, p=0.003$, box plots in Figure 4). This finding suggested that OT might strengthen the scaffolding effect of DDMNs on PN.

Generally, there was less correlation of the RSNs with the task-state network in the OT group. 

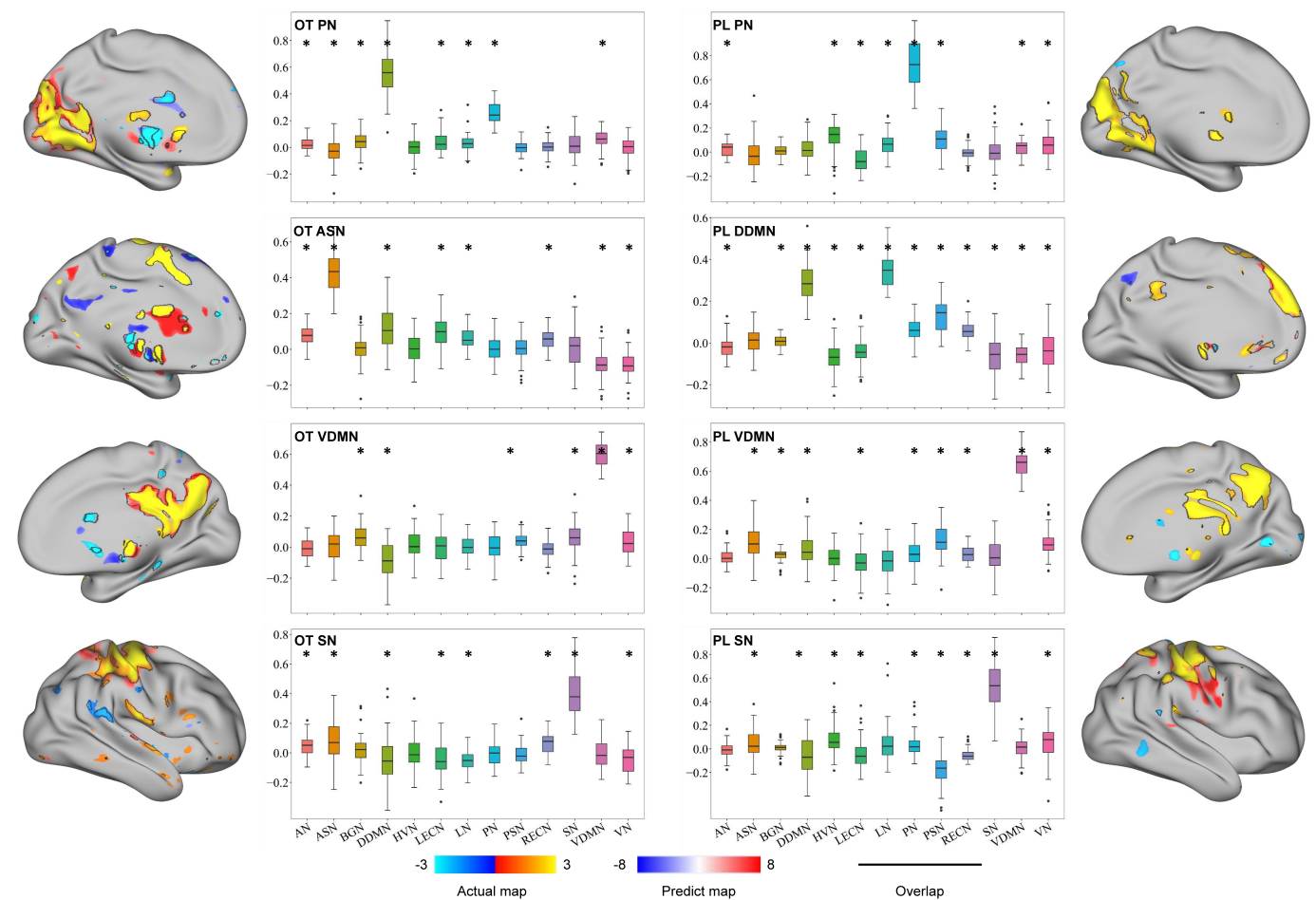

Figure 4: Predicting individual task activation maps. Beta coefficients(vertical coordinates) averaged across ICs that belong to one brain network indicate the contribution of the resting-state networks in predicting task-state features. For the most of features, the network's self-predictive power was the strongest, except for the PN under the OT group. The brain plots show actual and predicted task contrast maps for all features that are close to our task in two subjects (one for the OT group and one for the PL group). ${ }^{*} p_{F D R}<0.05$.

We used the ICs from rsfMRI data to forecast the four task-state ICs and got four prediction maps from each participant after model construction. We measured the recovery performance by comparing the predicted maps to the actual maps. The actual and predicted maps of the main regions of each IC had a significant rate of overlap (Figure 4). Our approach was also able to detect inter-subject variability in both groups. The model correctly recovered activation in areas that had no expression on average(Figure 5). As a result, we investigated the individual variation of network interactions across resting and task states. The subsection "Model performance and inter-subject variation" goes into more detail about the predictive accuracy and individual variation. 


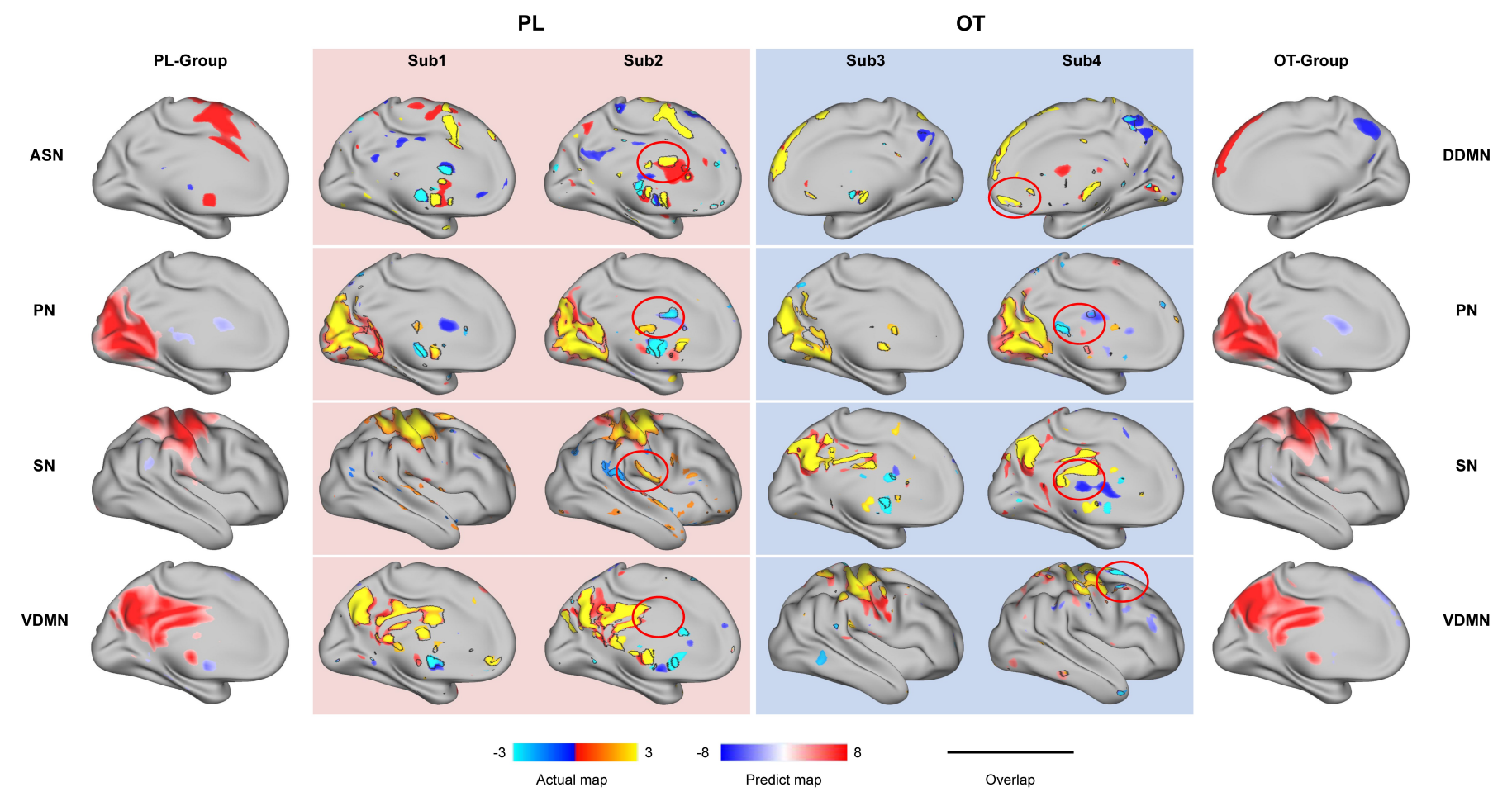

Figure 5: Personalized predictive maps. In both groups, our model showed the ability to detect inter-subject variability. The brain maps with white context indicate the group-averaged activation maps of networks, and the other four columns with color context show the actual and predicted activations of two subjects in one group (OT or PL). Although the main region was similar to the group activation, the model captured the specific clusters that were not showed in the group.

\subsection{Model performance and the inter-subject variation}

Two indices were used to test the model: The first was the coefficient of determination $R^{2}$, which represents how many variations could be explained by the models. Although the networks for prediction (resting-state ICs) and the modeling process were the same, we found that the variation of different task ICs explained by resting ICs varied greatly. Interestingly, the models performed the best when interpreting $\operatorname{DDMN}\left(R^{2}=0.39\right)$ but worst when interpreting $\operatorname{ASN}\left(R^{2}=0.23\right)$. Besides, no significantly differences in the interpretability of the same brain network was were found between groups (SI Figure 2, Table 2).

Another index is the prediction accuracy (see "Model test"). We examined whether the two methods (see "Model test") yielded different results. In most situations, the SM method performed better than OM, except for VDMN in the PL group and ASN in the OT group (Figure 6A, SI Table 1). Additionally, only PN showed significantly higher accuracy in the OT group than in the PL group in both methods (Figure 6A, SM: $t(58)=2.79, p=0.007$; OM: $t(58)=2.96, p=0.004$ ). 
Table 2: Coefficients of determination.

\begin{tabular}{lcc}
\hline \hline & \multicolumn{2}{c}{ Mean R square std } \\
\hline PL DDMN & 0.39 & 0.09 \\
PL PN & 0.31 & 0.10 \\
PL VDMN & 0.32 & 0.08 \\
PL SN & 0.24 & 0.10 \\
OT ASN & 0.23 & 0.07 \\
OT PN & 0.33 & 0.10 \\
OT VDMN & 0.33 & 0.07 \\
OT SN & 0.23 & 0.07 \\
\hline
\end{tabular}

Note: Means and standard deviations results of the coefficients of determination.

Additionally, compared to the PL group, there was a smaller difference in the accuracy between

Table 3: Predictive accuracy of others

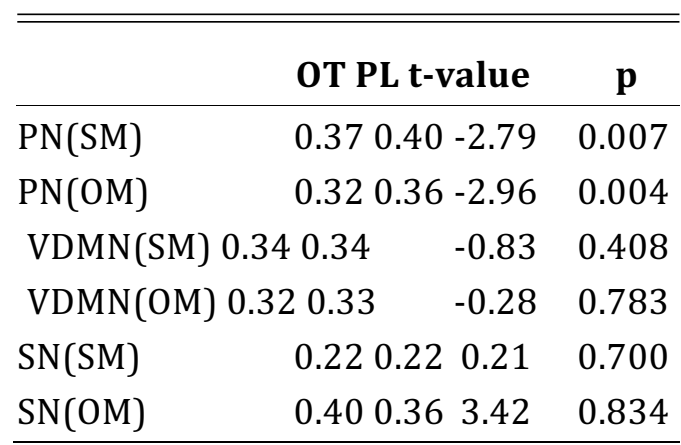

Note: Means, and two-tailed t-test (direction: OT - PL) results of the comparisons on predictive accuracy.

predicting own-task and predicting other-task activation maps in the OT group. Both methods showed that only PN displayed a significantly higher accuracy in predicting other's task maps in the OT group than in the PL group (Table 3, Figure 6A). The difference in the accuracy of PN indicated in the OT group was lower than that in the PL group when performing the OM method $(t(57)=-2.55, p=0.014$, Figure 6B). Meanwhile, SN and VDMN also showed this trend while the difference was not significant. These results suggest that OT may reduce inter-individual differences in PN. However, this effect was not observed when SM was used. 

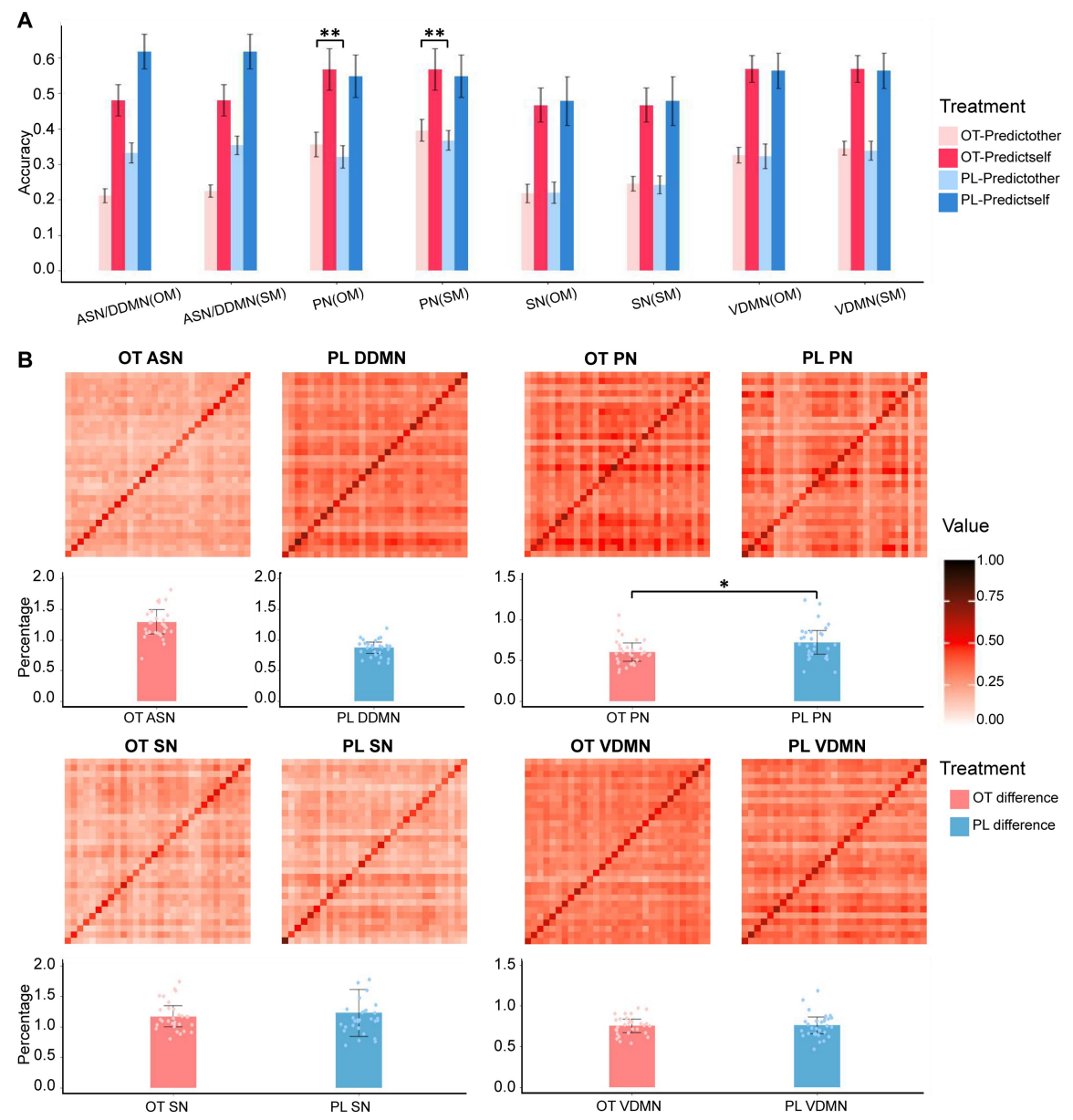

Figure 6: Performance of the model and specificity of the individual predictions. A) This figure shows the accuracy of using one's own model (SM) and others' models (OM) to predict one's own (deep color) and others' task-evoked network activation (light color) for both conditions. B) Pearson correlation matrix between actual (columns) and predicted activations (rows). The elements on the diagonal represent the accuracy of self-prediction, while those extradiagonal elements show the other-prediction accuracy. The bar plot, which was produced by the method using OM, indicates the difference between the accuracy of using participants' resting-state data to predict their task activation and predict others' activation (as a percentage relative to the average accuracy of predicting others).

\section{Discussion}

To the best of our knowledge, although the effect of OT on neural activity in both resting and task states has been well discussed, this work is the first study to investigate how OT affects the brain network activation and the relationships of these networks across resting and task states. In addition to confirming the effect of OT on brain networks during both resting state and face perception tasks, we also assessed its impact on the scaffolding mechanism of RSNs for task-evoked 
networks activation. Our study provided four promising preliminary results. First, there was a decreased activation over the DDMN for the OT group in the resting state. Second, compared to the PL group, there was a decreased functional connectivity between PN and DDMN, and PN and VDMN in the OT group as well. Third, in the OT group, the resting-state activity of the DDMN showed the largest predictive power for the task-evoked brain activity of PN, but not for the PL group. Finally, OT reduced self-other differences in PN when predicting task activation. Overall, our findings provide new evidence for the dynamic and interactive modulation of brain-wide networks by OT.

\subsection{OT effects on the sub-networks of DMN in resting and task state}

Although studies have discussed the modulation of DMN activity by 0T[54, 55], its effects on the sub-networks across rest to task states are still unrevealed. Its effects on the sub-networks across resting and task states are still unknown. Here, we utilized a more sophisticated template to define the networks, such as dividing DMN into DDMN and VDMN[40]. Our findings showed decreased DDMN activation in the resting states in the OT group, while showing no effect on VDMN. Previous studies have reported that the DDMN (including the MPFC and PCC) is involved in introspection about mental states and the evaluation of emotional valence, while VDMN (including the retrosplenial cortex and medial temporal lobe) is associated with memory-based reconstruction $[41,42]$. Thus, one possible explanation for our finding is that OT may have no impact on memory reconstruction processing but affects the emotional facets of human experience. Another possibility is that the effect of OT depends on the task. Our tasks focused on the selfrecognizing process, which may correlate with individual subjective feelings instead of memory reconstruction.

The precuneus, which is a brain region in the DMN, is also a crucial region in the PN. The present findings show decreased PN activation at rest in the OT group, whereas no group difference was observed for the task state. The precuneus plays a key role in mentalizing circuits[56, 57, 58], and self-reference processing[59]. Consistent with this idea and the knowledge of OT's effect on mentalizing networks[16], our results confirm the effect of OT on mentalizing or self-reference processing during resting. Based on the results described above that illustrate our first hypothesis on DMN, we infer that OT modulates self-evaluation, particularly the emotional component in this processing.

\subsection{DDMN has the highest predictive power for task-evoked activity of PN for the OT group}

Studies have shown a close relationship between $\mathrm{PN}$ and DMN, but with mixed results regarding whether PN is part of the $\operatorname{DMN}[60,61]$. Indeed, some studies showed that the precuneus is a functional core of the $\operatorname{DMN}[44,43]$. However, recent researches do not support this idea and indicate that PN is distinct from the DMN[43]. A meta-analysis of task-based fMRI and resting-state functional connectivity studies identified the PN (also called 'parietal memory network') as broadly involved in the formation of memory and personal experience of novelty[43]. Another fMRI study demonstrated the distinct functional roles of PN and DMN in processing context-rich 
information[44]. Our findings may add another piece of evidence on the overlap or interaction between PN and DMN.

In our results, we found that PN was negatively correlated DDMN and VDMN during the task regardless of whether it was affected by OT. The functional connectivity between PN and DDMN at rest was reversed in the OT group compared to that in the PL group (see Figure 3A, SI. Figure 1). Additionally, DDMN at rest had a stronger predictive power for PN in the task-state than resting PN itself in the OT group, but no significant predictive power in the PL group(Figure 4). This was the only case in which the network's performance at rest was not the best predictor of its activation during the task. Together, these results suggest that network dynamic changes depend on drug administration, for example, OT changes the way in which DDMN working in the task. In the PL group, DDMN plays a direct role during the task. In the OT group, DDMN participate in the task by modulating the activation of the $\mathrm{PN}$, rather than acting directly.

\subsection{OT reduced individual difference in PN prediction}

Our study provides two results regarding the impact of OT on individual variations in PN activation in both the resting and task states. The first one shows that OT may reduce the predictability of PN activation when using the Big-Five scale scores to predict the activity of PN (Figure 2B). The second one is reflected in the process of using rsfMRI data to predict the networks' expression in the task state. The result indicates that the self-other difference (i.e., the distinction between using subject X's resting-state data to predict X's task data and to predict subject Y's task data) of PN is significantly decreased by OT (Figure 6B). These findings are likely opposite to previous studies, which suggest that the impact of OT on people displays vast individual variability $[62,34,52,63]$. For instance, some studies reported that brain activation was significantly correlated with personal characteristics in the OT group, while the correlation was not significant in the PL group[34, 52, 63]. We proposed that the reduced individual variation makes the correlations between personal characteristics and brain activity easier to be observed. Notably, our resting/task-state prediction of the two groups focuses more on task brain prediction rather than individual differences per se. This may provide new insights for understanding the mechanism by which OT affects the human brain activity during tasks depending on the brain activity during the resting state.

In the OT group, we found a smaller self-other difference when predicting the task network's activation, which is only significant in PN. This may be due to the specific role of PN in our selfother face perception task. Andrea E. Cavanna and Trimble (2006) described a series of studies in which the precuneus is activated more for self-relevant than self-irrelevant personal traits[64, 65, $66]$, and more for a first-person than a third-person perspective $[67,58,68]$. Therefore, when the subjects recognize whether a face stimulus is similar to the face of the subjects themselves, 0T may have the greatest impact on the most important brain network, PN. Thus, it provides further evidence that task-evoked and connectivity data are essential to study the resting-task predictable networks. 


\section{Limitation and future direction}

The present study provides the first evidence that OT modulates the resting-task brain network with the self-other face perception task. It remains unknown whether our results can be generalized to other social tasks. In addition, the reason for the different networks displaying distinct predictability in our study remains unknown. Additionally, the sample size and the fact that our sample consisted of only males may also limit the generalizability of our results. For example, we cannot investigate all profiles of the OT effects on behavioral and brain networks that can be predicted by resting state, with one representative task. Perhaps, different types of tasks (e.g., social tasks and cognitive tasks) may result in specific OT effects on the resting-task brain prediction. Future studies are required to test the correlations of resting and task brain networks after OT administration and with multiple-task settings.

Another topic worth exploring is the consistency between people's subjective perceptions and their brain activity during different states. Accumulated evidence suggests that OT increases prosocial behavior[35, 69, 70], and our findings indicate that individual differences in networks are decreased in the OT group. Previous studies regarding the brain-heart interaction have reported that information flows more from the heart to the brain during a relaxed state such as sleep[71], but more information flows from the brain to the heart as the consciousness arousal level increases[72]. Other studies showed higher brain activation synchronism when people better understand others[73, 74]. The brain activity patterns of friends are more similar than those of strangers $[75,76]$. Since all these findings demonstrated individual insight into their neural system, does this consistency generalize to the prediction process? For example, whether the accuracies of individuals in the resting state predicting their performance in a task are consistent with the predictability of their task brain activity using their rsfMRI? What brain regions or networks display more consistency with subjective human perceptions? What factors could affect this consistency? We believe that our study may inspire future research regarding factors such as cue presentation sequence, moods, and social information, contributing to enhancing resting-task network prediction.

\section{Conclusion}

In summary, we observed three main results: 1) DDMN was decreased in the OT group in both resting; 2) the activity of DDMN in the resting state has the largest predictive power for the taskevoked activation of PN only for the OT group; and 3) as predicting task activation, OT reduces the self-other difference of PN. We believe these findings together reflect OT's distinct influence on diverse brain networks across different states and their relationships. The current study elucidated the mechanism by which OT affects the scaffolding mechanism of networks in the resting state to task activation. 


\section{Acknowledgements}

This work is funded by Natural Science Foundation of Guangdong Province (2021A1515012509), SRG of University of Macau (SRG2020-00027-ICI), Shenzhen-Hong KongMacao Science and Technology Innovation Project(SGDX 2020110309280100), Science and Technology Development Fund(FDCT) of Macau (0127/2020/A3), National Natural Science Foundation of China (NSFC) (U1736125), the Major Project of National Social Science Foundation (19ZDA363), the Beijing Municipal Science and Technology Commission (Z151100003915122), and Open Research Fund of the State Key Laboratory of Cognitive Neuroscience and Learning(CNLYB2002).

\section{References}

[1] C. G. DeYoung, J. B. Hirsh, M. S. Shane, X. Papademetris, N. Rajeevan, J. R. Gray, Testing predictions from personality neuroscience: Brain structure and the big five, Psychological science 21 (2010) 820-828.

[2] C. G. DeYoung, Personality neuroscience and the biology of traits, Social and Personality Psychology Compass 4 (2010) 1165-1180.

[3] S. Markett, C. Montag, M. Reuter, Network neuroscience and personality, Personality Neuroscience 1 (2018).

[4] M. C. Meyer, E. S. van Oort, M. Barth, Electrophysiological correlation patterns of resting state networks in single subjects: a combined eeg-fmri study, Brain topography 26 (2013) 98-109.

[5] T. O. Laumann, E. M. Gordon, B. Adeyemo, A. Z. Snyder, S. J. Joo, M.-Y. Chen, A. W. Gilmore, K. B. McDermott, S. M. Nelson, N. U. Dosenbach, et al., Functional system and areal organization of a highly sampled individual human brain, Neuron 87 (2015) 657-670.

[6] R. Kong, J. Li, C. Orban, M. R. Sabuncu, H. Liu, A. Schaefer, N. Sun, X.-N. Zuo, A. J. Holmes, S. B. Eickhoff, et al., Spatial topography of individual-specific cortical networks predicts human cognition, personality, and emotion, Cerebral cortex 29 (2019) 2533-2551.

[7] M. W. Cole, T. Ito, C. Cocuzza, R. Sanchez-Romero, The functional relevance of task-state functional connectivity, Journal of Neuroscience 41 (2021) 2684-2702.

[8] J. M. Shine, M. Breakspear, P. T. Bell, K. A. E. Martens, R. Shine, O. Koyejo, O. Sporns, R. A. Poldrack, Human cognition involves the dynamic integration of neural activity and neuromodulatory systems, Nature neuroscience 22 (2019) 289-296. 
[9] I. Tavor, O. P. Jones, R. B. Mars, S. Smith, T. Behrens, S. Jbabdi, Task-free mri predicts individual differences in brain activity during task performance, Science 352 (2016) 216-220.

[10] A. E. Reineberg, J. R. Andrews-Hanna, B. E. Depue, N. P. Friedman, M. T. Banich, Restingstate networks predict individual differences in common and specific aspects of executive function, Neuroimage 104 (2015) 69-78.

[11] G. Pezzulo, M. Zorzi, M. Corbetta, The secret life of predictive brains: what's spontaneous activity for?, Trends in Cognitive Sciences (2021).

[12] L. Pang, H. Li, Q. Liu, Y. Luo, D. Mobbs, H. Wu, Resting-state functional connectivity of social brain regions predicts motivated dishonesty (2021).

[13] M. L. Elliott, A. R. Knodt, M. Cooke, M. J. Kim, T. R. Melzer, R. Keenan, D. Ireland, S. Ramrakha, R. Poulton, A. Caspi, et al., General functional connectivity: Shared features of resting-state and task fmri drive reliable and heritable individual differences in functional brain networks, Neuroimage 189 (2019) 516-532.

[14] M. Horta, M. Ziaei, T. Lin, E. C. Porges, H. Fischer, D. Feifel, R. N. Spreng, N. C. Ebner, Oxytocin alters patterns of brain activity and amygdalar connectivity by age during dynamic facial emotion identification, Neurobiology of aging 78 (2019) 42-51.

[15] K. Brodmann, O. Gruber, R. Goya-Maldonado, Intranasal oxytocin selectively modulates largescale brain networks in humans, Brain connectivity 7 (2017) 454-463.

[16] H. Wu, X. Liu, C. C. Hagan, D. Mobbs, Mentalizing during social interaction: A four component model, Cortex 126 (2020) 242-252.

[17] Y. Wang, R. Wang, H. Wu, The role of oxytocin in modulating self-other distinction brain: a pharmacological fmri study, bioRxiv (2021).

[18] R. Hurlemann, A. Patin, O. A. Onur, M. X. Cohen, T. Baumgartner, S. Metzler, I. Dziobek, J. Gallinat, M. Wagner, W. Maier, et al., Oxytocin enhances amygdala-dependent, socially reinforced learning and emotional empathy in humans, J. Neurosci. 30 (2010) 4999-5007.

[19] D. Scheele, A. Wille, K. M. Kendrick, B. Stoffel-Wagner, B. Becker, O. Güntürkün, W. Maier, R. Hurlemann, Oxytocin enhances brain reward system responses in men viewing the face of their female partner, Proceedings of the National Academy of Sciences 110 (2013) 2030820313.

[20] F. Xin, F. Zhou, X. Zhou, X. Ma, Y. Geng, W. Zhao, S. Yao, D. Dong, B. B. Biswal, K. M. Kendrick, et al., Oxytocin modulates the intrinsic dynamics between attention-related largescale networks, Cerebral Cortex 31 (2021) 1848-1860. 
[21] R. Adolphs, The social brain: neural basis of social knowledge, Annual review of psychology 60 (2009) 693-716.

[22] L. F. Barrett, A. B. Satpute, Large-scale brain networks in affective and social neuroscience: towards an integrative functional architecture of the brain, Current opinion in neurobiology 23 (2013) 361-372.

[23] C. D. Frith, The social brain?, Philosophical Transactions of the Royal Society B: Biological Sciences 362 (2007) 671-678.

[24] D. A. Stanley, R. Adolphs, Toward a neural basis for social behavior, Neuron 80 (2013) 816826.

[25] A. Maier, D. Scheele, F. B. Spengler, T. Menba, F. Mohr, O. Güntürkün, B. Stoffel-Wagner, T. M. Kinfe, W. Maier, S. S. Khalsa, et al., Oxytocin reduces a chemosensory-induced stress bias in social perception, Neuropsychopharmacology 44 (2019) 281-288.

[26] M. C. Freitas-Ferrari, J. E. Hallak, C. Trzesniak, A. Santos Filho, J. P. Machado-de Sousa, M. H. N. Chagas, A. E. Nardi, J. A. S. Crippa, Neuroimaging in social anxiety disorder: a systematic review of the literature, Progress in Neuro-Psychopharmacology and Biological Psychiatry 34 (2010) 565-580.

[27] S. Zheng, D. Punia, H. Wu, Q. Liu, Graph theoretic analysis reveals intranasal oxytocin induced network changes over frontal regions, Neuroscience 459 (2021) 153-165.

[28] S. Zheng, Z. Liang, Y. Qu, Q. Wu, H. Wu, Q. Liu, Kuramoto model based analysis reveals oxytocin effects on brain network dynamics, arXiv preprint arXiv:2105.08288 (2021).

[29] X. Jiang, X. Ma, Y. Geng, Z. Zhao, F. Zhou, W. Zhao, S. Yao, S. Yang, Z. Zhao, B. Becker, et al., Intrinsic, dynamic and effective connectivity among large-scale brain networks modulated by oxytocin, BioRxiv (2020).

[30] M. Mennes, C. Kelly, X.-N. Zuo, A. Di Martino, B. B. Biswal, F. X. Castellanos, M. P. Milham, Interindividual differences in resting-state functional connectivity predict task-induced bold activity, Neuroimage 50 (2010) 1690-1701.

[31] M. W. Cole, T. Ito, D. S. Bassett, D. H. Schultz, Activity flow over resting-state networks shapes cognitive task activations, Nature neuroscience 19 (2016) 1718-1726.

[32] D. Bzdok, G. Varoquaux, O. Grisel, M. Eickenberg, C. Poupon, B. Thirion, Formal models of the network co-occurrence underlying mental operations, PLoS computational biology 12 (2016) e1004994. 
[33] M. W. Cole, D. S. Bassett, J. D. Power, T. S. Braver, S. E. Petersen, Intrinsic and task-evoked network architectures of the human brain, Neuron 83 (2014) 238-251.

[34] E. E. Hecht, D. L. Robins, P. Gautam, T. Z. King, Intranasal oxytocin reduces social perception in women: neural activation and individual variation, Neuroimage 147 (2017) 314-329.

[35] J. A. Bartz, J. Zaki, N. Bolger, K. N. Ochsner, Social effects of oxytocin in humans: context and person matter, Trends in cognitive sciences 15 (2011) 301-309.

[36] C. K. De Dreu, L. L. Greer, M. J. Handgraaf, S. Shalvi, G. A. Van Kleef, M. Baas, F. S. Ten Velden, E. Van Dijk, S. W. Feith, The neuropeptide oxytocin regulates parochial altruism in intergroup conflict among humans, Science 328 (2010) 1408-1411.

[37] R. Zhu, C. Liu, T. Li, Z. Xu, B. Fung, C. Feng, H. Wu, Y. Luo, L. Wang, Intranasal oxytocin reduces reactive aggression in men but not in women: A computational approach, Psychoneuroendocrinology 108 (2019) 172-181.

[38] J. R. Andrews-Hanna, J. S. Reidler, J. Sepulcre, R. Poulin, R. L. Buckner, Functionalanatomic fractionation of the brain's default network, Neuron 65 (2010) 550-562.

[39] J. S. Damoiseaux, C. Beckmann, E. S. Arigita, F. Barkhof, P. Scheltens, C. Stam, S. Smith, S. Rombouts, Reduced resting-state brain activity in the "default network" in normal aging, Cerebral cortex 18 (2008) 1856-1864.

[40] W. R. Shirer, S. Ryali, E. Rykhlevskaia, V. Menon, M. D. Greicius, Decoding subject-driven cognitive states with whole-brain connectivity patterns, Cerebral cortex 22 (2012) 158-165.

[41] S. Lee, T. Parthasarathi, J. W. Kable, The ventral and dorsal default mode networks are dissociably modulated by the vividness and valence of imagined events, Journal of Neuroscience 41 (2021) 5243-5250.

[42] J. E. Chen, G. H. Glover, M. D. Greicius, C. Chang, Dissociated patterns of anti-correlations with dorsal and ventral default-mode networks at rest, Human brain mapping 38 (2017) 24542465.

[43] A. W. Gilmore, S. M. Nelson, K. B. McDermott, A parietal memory network revealed by multiple mri methods, Trends in cognitive sciences 19 (2015) 534-543.

[44] Z. Deng, J. Wu, J. Gao, Y. Hu, Y. Zhang, Y. Wang, H. Dong, Z. Yang, X. Zuo, Segregated precuneus network and default mode network in naturalistic imaging, Brain Structure and Function 224 (2019) 3133-3144. 
[45] V. D. Calhoun, K. A. Kiehl, G. D. Pearlson, Modulation of temporally coherent brain networks estimated using ica at rest and during cognitive tasks, Human brain mapping 29 (2008) 828838.

[46] S. M. Platek, D. M. Raines, G. G. Gallup Jr, F. B. Mohamed, J. W. Thomson, T. E. Myers, I. S. Panyavin, S. L. Levin, J. A. Davis, L. C. Fonteyn, et al., Reactions to children's faces: Males are more affected by resemblance than females are, and so are their brains, Evolution and Human behavior 25 (2004) 394-405.

[47] S. M. Platek, F. B. Mohamed, G. G. Gallup Jr, Contagious yawning and the brain, Cognitive brain research 23 (2005) 448-452.

[48] S. M. Platek, S. M. Kemp, Is family special to the brain? an event-related fmri study of familiar, familial, and self-face recognition, Neuropsychologia 47 (2009) 849-858.

[49] S. Rachakonda, E. Egolf, N. Correa, V. Calhoun, Group ica of fmri toolbox (gift) manual, Dostupnez [cit 2011-11-5] (2007).

[50] J. Gaviria, G. Rey, T. Bolton, D. Van De Ville, P. Vuilleumier, Dynamic functional brain networks underlying the temporal inertia of negative emotions, bioRxiv (2021).

[51] F. Xin, X. Zhou, D. Dong, Z. Zhao, X. Yang, Q. Wang, Y. Gu, K. M. Kendrick, A. Chen, B. Becker, Oxytocin differentially modulates amygdala responses during top-down and bottom-up aversive anticipation, Advanced Science 7 (2020) 2001077.

[52] J. L. Alcorn III, C. E. Green, J. Schmitz, S. D. Lane, Effects of oxytocin on aggressive responding in healthy adult males (????).

[53] S. Chatterjee, A. S. Hadi, Influential observations, high leverage points, and outliers in linear regression, Statistical science (1986) 379-393.

[54] K. Brodmann, O. Gruber, R. Goya-Maldonado, Intranasal oxytocin selectively modulates largescale brain networks in humans, Brain Connectivity (2017) 454-463.

[55] H. Wu, C. Feng, X. Lu, X. Liu, Q. Liu, Oxytocin effects on the resting-state mentalizing brain network, Brain imaging and behavior (2020) 1-12.

[56] A. E. Cavanna, M. R. Trimble, The precuneus: a review of its functional anatomy and behavioural correlates, Brain 129 (2006) 564-583. 
[57] M. Schurz, J. Radua, M. Aichhorn, F. Richlan, J. Perner, Fractionating theory of mind: a metaanalysis of functional brain imaging studies, Neuroscience \& Biobehavioral Reviews 42 (2014) 9-34.

[58] K. Vogeley, P. Bussfeld, A. Newen, S. Herrmann, F. Happ'e, P. Falkai, W. Maier, N. J. Shah, G. R. Fink, K. Zilles, Mind reading: neural mechanisms of theory of mind and self-perspective, Neuroimage 14 (2001) 170-181.

[59] S. Whitfield-Gabrieli, J. M. Moran, A. Nieto-Castañón, C. Triantafyllou, R. Saxe, J. D. Gabrieli, Associations and dissociations between default and self-reference networks in the human brain, Neuroimage 55 (2011) 225-232.

[60] P. Fransson, G. Marrelec, The precuneus/posterior cingulate cortex plays a pivotal role in the default mode network: Evidence from a partial correlation network analysis, Neuroimage 42 (2008) 1178-1184.

[61] A. V. Utevsky, D. V. Smith, S. A. Huettel, Precuneus is a functional core of the default-mode network, Journal of Neuroscience 34 (2014) 932-940.

[62] R. Kumsta, M. Heinrichs, Oxytocin, stress and social behavior: neurogenetics of the human oxytocin system, Current opinion in neurobiology 23 (2013) 11-16.

[63] S. Koch, M. V. Zuiden, L. Nawijn, J. L. Frijling, D. J. Veltman, M. Olff, Intranasal oxytocin normalizes amygdala functional connectivity in posttraumatic stress disorder, Neuropsychopharmacology 41 (2016) 2041-2051.

[64] T. T. Kircher, C. Senior, M. L. Phillips, P. J. Benson, E. T. Bullmore, M. Brammer, A. Simmons, S. C. Williams, M. Bartels, A. S. David, Towards a functional neuroanatomy of self processing: effects of faces and words, Cognitive Brain Research 10 (2000) 133-144.

[65] T. W. Kjaer, M. Nowak, H. C. Lou, Reflective self-awareness and conscious states: Pet evidence for a common midline parietofrontal core, Neuroimage 17 (2002) 1080-1086.

[66] H. C. Lou, B. Luber, M. Crupain, J. P. Keenan, M. Nowak, T. W. Kjaer, H. A. Sackeim, S. H. Lisanby, Parietal cortex and representation of the mental self, Proceedings of the National Academy of Sciences 101 (2004) 6827-6832.

[67] H. E. Den Ouden, U. Frith, C. Frith, S.-J. Blakemore, Thinking about intentions, Neuroimage 28 (2005) 787-796.

[68] K. Vogeley, G. R. Fink, Neural correlates of the first-person-perspective, Trends in cognitive sciences 7 (2003) 38-42. 
[69] C. S. Carter, Oxytocin pathways and the evolution of human behavior, Annual review of psychology 65 (2014) 17-39.

[70] C. K. De Dreu, M. E. Kret, Oxytocin conditions intergroup relations through upregulated ingroup empathy, cooperation, conformity, and defense, Biological psychiatry 79 (2016) 165173.

[71] J. MIN, J. WANG, Analysis of sleep signals based on permutation symbolic transfer entropy (2017).

[72] A. Abukonna, X. Yu, C. Zhang, J. Zhang, Volitional control of the heart rate, International journal of psychophysiology 90 (2013) 143-148.

[73] E. Simony, C. J. Honey, J. Chen, O. Lositsky, Y. Yeshurun, A. Wiesel, U. Hasson, Dynamic reconfiguration of the default mode network during narrative comprehension, Nature communications 7 (2016) 1-13.

[74] A. Zadbood, J. Chen, Y. C. Leong, K. A. Norman, U. Hasson, How we transmit memories to other brains: constructing shared neural representations via communication, Cerebral cortex 27 (2017) 4988-5000.

[75] C. Parkinson, A. M. Kleinbaum, T. Wheatley, Similar neural responses predict friendship, Nature communications 9 (2018) 1-14.

[76] R. Hyon, Y. Youm, J. Kim, J. Chey, S. Kwak, C. Parkinson, Similarity in functional brain connectivity at rest predicts interpersonal closeness in the social network of an entire village, Proceedings of the National Academy of Sciences 117 (2020) 33149-33160. 